\title{
PREVALENCIA Y FACTORES ASOCIADOS CON SÍNTOMAS DEPRESIVOS EN ESTUDIANTES DE CIENCIAS DE LA SALUD DE UNA UNIVERSIDAD PRIVADA DE LIMA, PERÚ 2010*
}

\author{
Reneé Pereyra-Elías 1,2,a, Javier Ocampo-Mascaró1,2,a, Vera Silva-Salazar ${ }^{1, a}$, Eduardo Vélez-Segovia ${ }^{1,2, a}$,
} A. Daniel da Costa-Bullón ${ }^{1, a}$, Luis Miguel Toro-Polo ${ }^{1,2, a}$, Joanna Vicuña-Ortega 1,2,a

\begin{abstract}
RESUMEN
Introducción. Los síntomas depresivos en estudiantes de ciencias de la salud son comunes y podrían ser potencialmente perjudiciales. Objetivos. Determinar la prevalencia de síntomas depresivos y sus factores asociados en los estudiantes de la Facultad de Ciencias de la Salud de la Universidad Peruana de Ciencias Aplicadas, Lima (Perú), junio 2010. Materiales y métodos. Estudio analítico transversal; se realizó una encuesta previo consentimiento informado a 590/869 estudiantes. Para medir la variable de respuesta (síntomas depresivos) se usó la escala de Zung abreviada. Se empleó la regresión logística múltiple para evaluar los factores asociados, considerando un nivel de significancia de $p<0,05$. Resultados. La edad media fue 18,97 $\pm 2,45$ años; $71,1 \%$ fueron mujeres; $19,6 \%$ fueron migrantes y $62,5 \%$ fueron de la carrera de medicina. La prevalencia de síntomas depresivos fue 31,2\% en la población y de 33,6\% en estudiantes de medicina. No se encontró asociación en el análisis bivariado con el sexo, carrera de estudio, si ha repetido cursos, si vive solo o si es migrante $(p>0,05)$. En el análisis multivariado, se encontró asociación con la inconformidad de su rendimiento académico ( $\mathrm{OR}=2,13$; IC95\%:1,47-3,08), inconformidad con la situación económica actual $(\mathrm{OR}=1,93$; IC95\%:1,24-2,99) y vivir con un familiar externo a la familia nuclear (OR=1,62; IC95\%:1,07-2,45). Conclusión. Existe una alta prevalencia de síntomas depresivos en la población estudiada, especialmente en los alumnos de la carrera de medicina, siendo la inconformidad con el rendimiento académico, con su economía y vivir con un familiar externo a la familia nuclear, factores asociados que podrían tomarse en cuenta para trabajar programas preventivos.
\end{abstract}

Palabras clave. Depresión; Estudiantes del Área de la Salud; Estudiantes de Medicina; Perú (fuente: DeCS BIREME).

\section{PREVALENCE AND ASSOCIATED FACTORS WITH DEPRESSIVE SYMPTOMS IN HEALTH SCIENCES STUDENTS FROM A PRIVATE UNIVERSITY IN LIMA, PERU 2010}

\begin{abstract}
Introduction. Depressive symptoms in health sciences students are common, these might be potentially detrimental. Objectives. To determine the prevalence of depressive symptoms and its associated factors in students from the Health Sciences Faculty of the Universidad Peruana de Ciencias Aplicadas in Lima (Peru), June 2010. Materials and methods. Cross-sectional analytic study; a pre-consented survey was applied to the population 590/869 students. Zung's abbreviated scale was used to measure depressive symptoms. To evaluate de associated factors, logistic regression was used, $p<0.05$ was considered statistically significant. Results. The mean age was $18.97 \pm 2.45$ years and $71.1 \%$ were women, $19.6 \%$ were migrants and $62.5 \%$ were medical students. The prevalence of depressive symptoms was $31.2 \%$ in the whole population and in medical students was $33.6 \%$. Depressive symptoms were not associated in bivariate analysis with sex, career, having failed a course, living alone or being a migrant $(p>0.05)$. In the multivariate analysis, significant statistical association was found between depressive symptoms and dissatisfaction with the own academic performance $(\mathrm{OR}=2.13 \mathrm{Cl}$ (95\%1.47-3.08), dissatisfaction with the current economic status (OR=1.93 Cl95\%1.24-2.99) and living with a relative external to the nuclear family (OR=1.62 Cl95\%1.07-2.45). Conclusion. A high prevalence of depressive symptoms was found, especially in medical students; being dissatisfaction with academic performance, economic status and living with a relative external to the nuclear family associated factors that could be taken into account in order to build preventive programs.
\end{abstract}

Key words. Depression; Students, Health Occupations; Medical Students; Peru (source: MeSH NLM).

Escuela de Medicina, Universidad Peruana de Ciencias Aplicadas, Lima, Perú.

Sociedad Científica de Estudiantes de Medicina de la Universidad Peruana de Ciencias Aplicadas (SOCIEMUPC), Lima, Perú.

Estudiante de Medicina.

Este trabajo fue presentado al XXIV Congreso Científico Nacional de Estudiantes de Medicina, Arequipa 2010, organizado por la Sociedad Científica Médica Estudiantil Peruana (SOCIMEP)

Recibido: 30-08-10 Aprobado: 01-12-10 


\section{INTRODUCCIÓN}

La depresión es un desorden afectivo multifactorial que se puede manifestar como una serie de síntomas tanto físicos como psicológicos que suelen obstaculizar la vida diaria y el desempeño normal del individuo que lo padece ${ }^{(1,2)}$, y es una de las causas más importantes de discapacidad en el mundo ${ }^{(2)}$.

Para detectar síntomas depresivos, se puede recurrir a cuestionarios autoaplicados, los cuales son interpretados de acuerdo con diversas escalas ya estandarizadas (3). Sin embargo, para dar un diagnóstico definitivo de depresión, diversos autores afirman que es necesario recurrir a una evaluación clínica, ya que las escalas de depresión no evalúan todos los criterios clínicos, sino que priorizan aspectos característicos, que puede no sean suficientes para definir dicha enfermedad ${ }^{(4)}$.

Estudiar una carrera de ciencias de la salud se caracteriza por una exigencia académica elevada ${ }^{(5)}$. Investigaciones anteriores exponen que la mayor exigencia está asociada con mayores niveles de estrés. Un estudio realizado en estudiantes de carreras relacionadas con la salud como medicina, odontología, enfermería y psicología mostró un nivel elevado de prevalencia de estrés académico $(36,3 \%)^{(6)}$. El sentirse bajo presión por los exámenes, falta de apoyo familiar, experimentar presión por parte de sus profesores y tener dificultades económicas y relacionales, son características que se ha demostrado que están asociadas con una sintomatología depresiva y ansiosa en estudiantes de ciencias de la salud ${ }^{(6-9)}$.

Una circunstancia que puede llevar al desarrollo de síntomas depresivos es la migración, ya que esta implica un suceso de cambios que llevan al individuo a un proceso de adaptación a una nueva realidad, el cual puede conllevar a problemas como sufrir estrés o enfermedad mental ${ }^{(10-12)}$. Por otro lado, la falta de apoyo social (amical y familiar) y, nuevamente, los altos niveles de estrés, pueden devenir en depresión ${ }^{(11)}$.

Otro factor relacionado con la presencia de trastornos depresivos es el bajo nivel socioeconómico, ya que este puede implicar ciertos grados de tristeza, niveles altos de estrés, incertidumbre, entre otras características que podrían llevar al desarrollo de síntomas depresivos ${ }^{(13)}$. Se ha encontrado también una relación entre la calidad de la relación familiar y depresión, mostrando un aumento de depresión a medida que la calidad de la relación familiar disminuía, como al encontrarse lejos del núcleo familiar o carecer de apoyo afectivo emocional por parte de este ${ }^{(7,9)}$.

Por otro lado, los síntomas y trastornos depresivos podrían devenir en consecuencias negativas de índole académico, como el bajo rendimiento o la deserción en estudiantes universitarios ${ }^{(14,15)}$. Debido a esto, es necesario tomar las medidas adecuadas que permitan proteger la salud mental de la población estudiada.

La Universidad Peruana de Ciencias Aplicadas (UPC) ubicada en Lima tiene una elevada presencia $(18,3 \%)$ de estudiantes migrantes y no se tiene información previa sobre la salud mental de sus estudiantes, el objetivo fue determinar la prevalencia de síntomas depresivos y los factores asociados en estudiantes de ciencias de la salud de la UPC.

\section{MATERIALES Y MÉTODOS}

\section{DISEÑO Y LUGAR DE ESTUDIO}

Se realizó un estudio observacional, analítico de corte transversal en el mes de junio de 2010 en la Facultad de Ciencias de la Salud de la UPC, que cuenta con las carreras de Medicina, Odontología, Nutrición y Terapia Física. La Facultad de Ciencias de la Salud es reciente, sus alumnos de la carrera de medicina pertenecen del primer al cuarto año de estudios (no hay aún estudiantes cursando el internado), los estudiantes de odontología pertenecen del primer al segundo año, los de terapia física al primer año. Los estudiantes de nutrición son del primer al último año (sexto). El estudio fue aprobado por la Cátedra de Metodología de Investigación de la universidad.

\section{POBLACIÓN}

Se incluyó a todos los alumnos matriculados en el primer ciclo académico del 2010, a los cuales se les informó previamente de los objetivos del estudio y aquellos que estuvieron de acuerdo Ilenaron la encuesta. Los participantes fueron ubicados en las aulas de clase de aquellos cursos que abarcan casi a la totalidad de alumnos por carrera. Los encuestadores fueron los miembros del equipo investigador, quienes estuvieron presentes durante todo el tiempo que tomó el llenado de la encuesta (10 a 15 minutos) para resolver cualquier duda de los participantes. Fueron excluidas las personas que no aceptaron participar y quienes no llenaron las variables principales.

\section{INSTRUMENTO}

La encuesta fue anónima y autoadministrada. Constó de 27 preguntas con diversos apartados, los cuales incluyeron datos generales, datos sociodemográficos y factores asociados con síntomas depresivos (migración y otros). 
Para evaluar la presencia de síntomas depresivos se usó la escala de Zung abreviada (diez preguntas), la cual ha sido previamente validada en estudiantes de una universidad colombiana, tiene una sensibilidad de 0,92 y especificidad de 0,71 y con un punto de corte de 22 o más para el tamizaje de un Episodio Depresivo Mayor, usando como gold standard la entrevista clínica estructurada para los trastornos del eje I (SCID-I) del DSM-IV (16). Las afirmaciones contenidas en la escala de Zung se refirieron a los 15 días previos a la toma de la encuesta.

Se midió los siguientes factores asociados: migración, personas con las que vive el encuestado, conformidad con el rendimiento académico, conformidad con la situación económica propia, carrera y conformidad con la carrera elegida. Las variables de conformidad fueron medidas con una pregunta directa dicotómica (¿estás conforme con...?). La variable referida a las personas con quienes vive el encuestado fue medida por una pregunta de opción multiple para marcar.

\section{DEFINICIÓN DE VARIABLES}

Se definió como persona con síntomas depresivos al individuo que haya obtenido 22 puntos o más en la escala (16). Los estudiantes fueron agrupados según carrera en estudiantes de medicina y estudiantes de otras carreras, debido a que los estudiantes de medicina representan el $55 \%$ de la población.
La variable migración fue medida de distintas maneras debido a que no se encontró una definición estándar para usarla dentro de un contexto de investigación. Se usaron cinco definiciones, las cuales definen a una persona migrante como: aquella que haya vivido menos de dos años en Lima en sus cinco años previos al ingreso de la univerdad, aquella que haya vivido menos de cinco años en Lima, aquella que haya conluido sus estudios escolares fuera de Lima, aquella que, antes de ingresar a la universidad, haya residido en un lugar distinto a Lima y por último, aquella cuyo lugar de nacimiento sea diferente de Lima.

\section{ANÁLISIS DE DATOS}

Los resultados de las encuestas fueron digitalizados en una base de datos en Excel, luego del control de calidad se eliminaron las encuestas que no habían llenado en forma completa los ítems de la escala de Zung.

Se utilizó la prueba de $\mathrm{chi}^{2}$ (variables dicotómicas) o $t$ de Student (variables cuantitativas continuas con distribución normal) en el análisis bivariado para evaluar la asociación entre síntomas depresivos y las variables de exposición. Se consideró un $p<0,05$ como significativo. Las variables asociadas en el análisis bivariado fueron incluidas en un modelo de regresión logística múltiple, se generó las variables de interacción y se evaluó el modelo completo frente al reducido con la prueba de verosimilitud, finalmente se usó la prueba

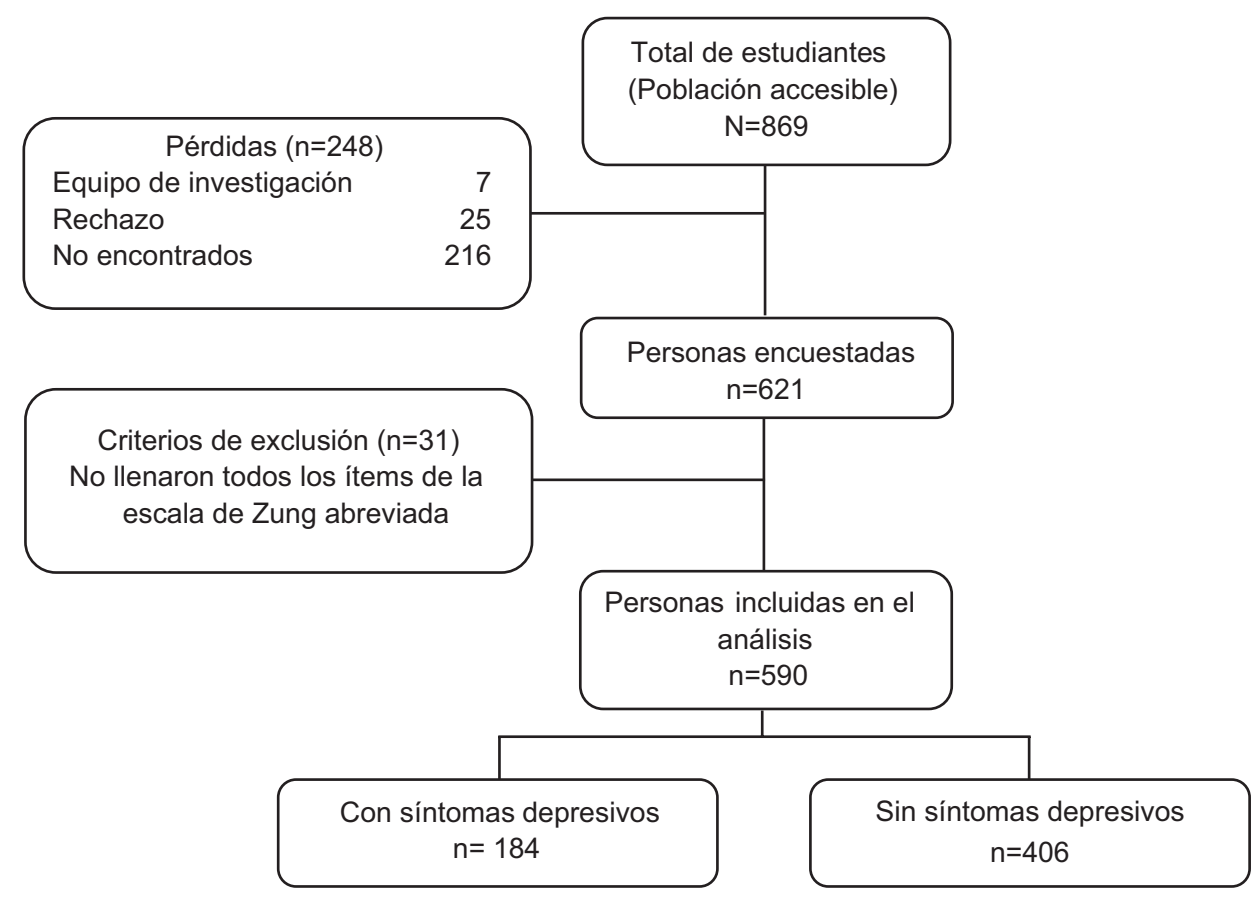

Figura 1. Flujograma de selección de participantes en el estudio. 
de bondad de ajuste (Hosmer Lemeshow) para evaluar la validez del modelo. El análisis de datos se realizó usando el paquete estadístico Stata 11.0.

\section{RESULTADOS}

De un total de 869 estudiantes de ciencias de la salud (incluyendo a las carreras de medicina, odontología, nutrición y terapia física), se encuestó a 621 personas (tasa de aceptación de 71,5\%), de las cuales 41 fueron eliminadas por problemas en el llenado, quedando 590 $(68 \%)$ encuestas incluidas (Figura 1).

\section{CARACTERÍSTICAS DE LA POBLACIÓN}

La edad promedio fue de $18,97 \pm 2,45$ años y el 71,1 $\%$ fueron mujeres. Se incluyó al $78,2 \%$ de personas pertenecientes a la carrera de Medicina y al $68,5 \%$ de las otras carreras (nutrición, odontología y terapia física).

Un porcentaje reducido de estudiantes vive solo $(4,4 \%)$, mientras que la mayoría vive con al menos un familiar: $75,9 \%$ con la madre; $64,9 \%$ con el padre; $69,3 \%$ con un hermano/a y $22,4 \%$ con otro familiar no perteneciente a la familia nuclear. El $97,8 \%$ está estudiando la carrera que quiere, $55,9 \%$ de los estudiantes no se encuentra conforme con su rendimiento académico y $80,7 \%$ percibe lo mismo respecto de su situación económica actual.

\section{SÍNTOMAS DEPRESIVOS Y FACTORES ASOCIADOS}

Se encontró una prevalencia de síntomas depresivos en la población total de 31,2\%. Dicha prevalencia fue mayor en los estudiantes de medicina $(33,6 \%$ ) y los de las otras carreras de la facultad presentaron una prevalencia de $26,5 \%(p>0,05)$.

Se encontró que los estudiantes que no están conformes con la carrera que estudian presentaron mayor prevalencia de síntomas depresivos que los que sí lo estaban ( $42 \%$ frente a $23 \%, p=0,039)$; así mismo, el estar inconformes con su situación económica se asoció con mayor prevalencia de síntomas depresivos (47\% frente a $28 \%, p<0,001)$. La edad, el sexo, el lugar de nacimiento, trabajar, vivir solo, vivir con la madre, haberse cambiado de carrera y haber desaprobado cursos durante la carrera no tienen asociación significativa con tener síntomas depresivos (Tabla 1).

Las variables en las que se encontró asociación significativa $(p<0,05)$ fueron incluidas en el modelo multivariado de regresión logística; sin embargo, la variable vivir con un hermano/a no fue incluida en el modelo final por perder su asociación y, con su inclusión,

Tabla 1. Factores asociados con la presencia de síntomas depresivos en estudiantes de las ciencias de la salud, Universidad Peruana de Ciencias Aplicadas, 2010.

\begin{tabular}{|c|c|c|c|c|c|}
\hline \multirow[t]{2}{*}{ Factores evaluados } & $\begin{array}{l}\text { Con síntomas } \\
\text { depresivos }\end{array}$ & $\begin{array}{l}\text { Sin síntomas } \\
\text { depresivos }\end{array}$ & \multirow[t]{2}{*}{ OR } & \multirow[t]{2}{*}{ IC $95 \%$} & \multirow[t]{2}{*}{$\mathbf{p}$} \\
\hline & n $(\%)^{*}$ & No $(\%)^{*}$ & & & \\
\hline Inconforme con su rendimiento académico & $108(59,0)$ & $149(37,3)$ & 2,43 & $1,67-3,53$ & $<0,001$ \\
\hline Inconforme con su situación económica & $53(29,0)$ & $60(14,9)$ & 2,32 & $1,49-3,61$ & $<0,001$ \\
\hline Vive con otros familiares $†$ & $54(29,3)$ & $78(19,4)$ & 1,73 & $1,23-2,63$ & 0,008 \\
\hline Vive con hermanos & $116(63,0)$ & $293(72,7)$ & 0,64 & $0,44-0,95$ & 0,018 \\
\hline Vive con padre & $110(59,8)$ & $273(67,9)$ & 0,70 & $0,48-1,03$ & 0,055 \\
\hline Estudia medicina & $124(70,1)$ & $245(62,5)$ & 1,40 & $0,94-2,10$ & 0,081 \\
\hline $\begin{array}{l}\text { Mantiene contacto regular con sus amigos fuera } \\
\text { de la Universidad }\end{array}$ & $161(87,5)$ & $363(90,1)$ & 0,77 & $0,43-1,40$ & 0,350 \\
\hline Varón & $50(27,3)$ & $126(31,0)$ & 0,84 & $0,55-1,25$ & 0,362 \\
\hline Vive solo & $9(4,9)$ & $17(4,2)$ & 1,16 & $0,45-2,83$ & 0,717 \\
\hline Migrante§ & $42(22,8)$ & $98(24,1)$ & 0,93 & $0,60-1,43$ & 0,729 \\
\hline Ha desaprobado cursos & $72(39,3)$ & $156(38,8)$ & 1,02 & $0,70-1,49$ & 0,902 \\
\hline
\end{tabular}

* Porcentaje calculado considerando el denominador a la presencia de la variable evaluada y en el denominador al número de personas con o sin síntomas depresivos, según correspondía.

$\dagger$ Vive con otros familiares diferentes a su familia nuclear (padres y hermanos).

‡ Comparado con el grupo de estudiantes de otras ciencias de la salud (odontología, nutrición y tecnología médica) como un solo grupo.

$\S$ Los resultados mostrados son los que se encontraron con la siguiente definición de migrante: persona que no ha vivido por lo menos cinco años en Lima sin contar los años de estudios universitarios; sin embargo con las otras cuatro definiciones trabajadas no se encontró asociación. 
el modelo no tenía un buen ajuste (Hosmer Lemeshow; $\mathrm{p}=0,12)$. El modelo final incluyó a tres variables y tuvo un buen ajuste (Hosmer Lemeshow; $p=0,78$ ). El tener una percepción negativa (disconformidad) respecto del propio rendimiento académico $(\mathrm{OR}=2,13$; IC95\% 1,47$3,08)$, la disconformidad con la situación económica actual $(O R=1,93 ; \quad I C 95 \%$ 1,24-2,99) y vivir con otro familiar (OR=1,62 IC95\% 1,07-2,45) se comportaron como factores asociados con la presencia de síntomas depresivos,

\section{DISCUSIÓN}

Los resultados encontrados muestran una considerable prevalencia de síntomas depresivos en la población; especialmente, en la carrera de Medicina, cuya prevalencia es mayor que las otras carreras de Ciencias de la Salud (33,6\% frente a $26,5 \%)$. Esta prevalencia concuerda con las encontradas en diversos estudios que también evalúan la misma variable (Figura 2). Investigaciones realizadas en Medellín (Colombia) (7), Lima (Perú) ${ }^{(5)}$ y Cali (Colombia) ${ }^{(8)}$ mostraron una prevalencia de síntomas depresivos de 30,3; 29,6 y $36,5 \%$ respectivamente utilizando la escala de Zung. Otro estudio empleó el inventario de depresión de Beck en una universidad de Corrientes (Argentina) ${ }^{(9)}$ y reportó una prevalencia de $33,4 \%$. Otra investigación revisada, en la cual se encontró una prevalencia de síntomas depresivos menor a la hallada en el presente estudio, fue realizada en alumnos del quinto año de medicina de una universidad privada de Lima utilizando Escala de Psicopatología Depresiva (EPD) y la Escala de Depresión del Centro de Estudios Epidemiológicos (CES-D). Se reportó una prevalencia de $19 \%$. No obstante, la prevalencia de personas con probable trastorno depresivo mayor reportada en el estudio fue mucho menor (menos del 10\%) (17). En un estudio realizado por Osada et al., utilizando la escala de Goldberg para ansiedad y depresión en estudiantes de medicina de una universidad peruana, se reportó una prevalencia de $29,9 \%$ de sintomatología depresiva (18), lo cual es muy similar a lo encontrado en nuestra población.

Con estos hallazgos, podemos afirmar que aproximadamente tres de cada diez estudiantes de medicina presentan síntomas depresivos; probablemente, debido a que la carrera presenta constantes y mayores exigencias académicas que pueden afectar la salud mental del individuo. No se ha realizado una comparación de las prevalencias de síntomas depresivos en las otras carreras de ciencias de la salud debido a que no se encontró bibliografía actualizada al respecto. Al comparar nuestros resultados con el estudio en el cual se validó la escala utilizada ${ }^{(16)}$, que no incluyó estudiantes de medicina, se aprecia una gran diferencia en la prevalencia de tendencias depresivas entre estos dos grupos. La prevalencia en dicho estudio fue de $18,8 \%$, relativamente baja en comparación con la prevalencia del presente estudio $(33,6 \%)$.

Por otro lado, los factores asociados encontrados en el estudio (inconformidad con el rendimiento académico, con la situación económica y vivir con un familiar no perteneciente a la familia nuclear) no han sido encontrados en investigaciones previas.

En primer lugar, la conformidad con el rendimiento académico supone la percepción del estudiante respecto a ello, pudiendo ser este "bueno" o "malo" según los estándares particulares de cada individuo.

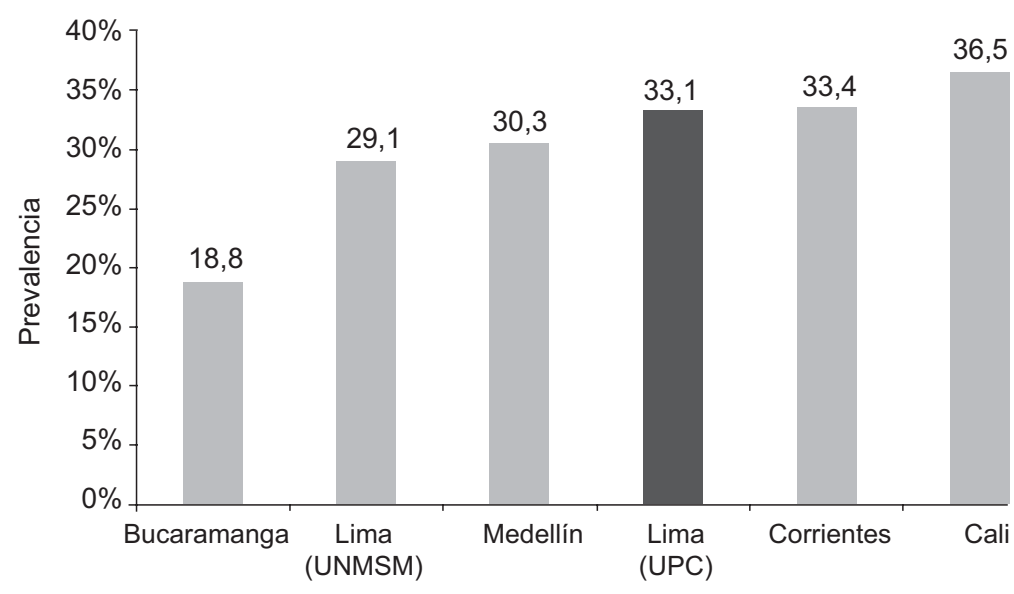

Figura 2. Comparación de prevalencias de síntomas depresivos en estudiantes de medicina de América Latina *.

* Los estudios usaron la escala de Zung para depresión (clásica), excepto el estudio en Corrientes, Argentina, que empleó la escala de Beck y el estudio de Bucaramanga, que trabajó con la versión abreviada de la escala de Zung.

UNMSM: Universidad Nacional Mayor de San Marcos. UPC: Universidad Peruana de Ciencias Aplicadas 
Es importante resaltar que se midió la variable "cursos desaprobados", la cual no tuvo asociación con los síntomas depresivos $(p=0,90)$. Por tanto, la variable de respuesta depende de la percepción o impresión del individuo en relación con su desempeño o rendimiento académico y no del resultado académico real. La otra pregunta que apeló a la percepción del individuo se refirió a la situación económica propia. Coincidentemente, ambas presentaron relación en el análisis multivariado. Estos factores pueden ser una fuente de estrés que eventualmente podría llevar a la depresión $(7,10,13)$.

Otro aspecto relacionado, de acuerdo al modelo multivariado, fue el vivir con otro familiar. Si bien los individuos que marcaron esta opción no necesariamente carecen de un miembro de la familia nuclear en su hogar, es probable que vivan con un familiar lejano y sin sus padres o hermanos, el cual es el caso de muchos migrantes. Es importante resaltar el papel de apoyo social que supone la familia, lo cual se evidencia notablemente en la influencia protectora de tener al padre o un hermano en el hogar ante el riesgo de presentar síntomas depresivos (7). No obstante, en el modelo multivariado, la importancia estadística de estas variables no fue suficiente para ser consideradas como factores protectores como lo hacía suponer el análisis bivariado.

En el caso de los migrantes (19,6\%), no se encontró asociación alguna con un riesgo aumentado de presentar síntomas depresivos. Aunque la revisión bibliográfica previa evidenciaba que el estrés aculturativo era un factor de riesgo para presentar tales síntomas ${ }^{(10-12)}$, es probable que las características de la población estudiada (nivel socioeconómico alto, oportunidad de estudiar en Lima) y otros factores, como el Programa de Acompañamiento Universitario brindado por la institución (para apoyar a los migrantes del interior del país), atenúen la influencia de la variable migración sobre el riesgo de presentar síntomas depresivos ${ }^{(7)}$.

Es importante mencionar que no se encontró asociación entre el sexo y la presencia de síntomas depresivos, a pesar de que hay estudios que afirman que las mujeres son más propensas a padecer trastornos depresivos (19). Probablemente, esto se debe a las características de la población de estudio, ya que las mujeres solteras, jóvenes y con estudios superiores completos no presentan diferencias de importancia en dicha prevalencia respecto de los hombres ${ }^{(19)}$.

Debido a que se realizó un censo, se encontraron algunas dificultades con respecto a la ubicación de todos los estudiantes de las distintas carreras encuestadas, ya que no se encontraban presentes en los salones al momento de la encuesta o no se podían hallar las aulas en donde se debió haber dictado la clase, a pesar de haber sido buscados en más de una oportunidad.

El estudio presenta algunas limitaciones, ya que no se pudo encuestar a la totalidad de la población, siendo menor el porcentaje de reclutamiento en estudiantes que no son de medicina. No se recabó información sobre las personas que no participaron del estudio, sin embargo, los estudiantes migrantes y mujeres de la Facultad son muy similares a los incluidos en el estudio; sin embargo, existe la posibilidad de que hayan diferencias en otras variables entre la población de estudio y los que no participaron; por ello, no se puede descartar la presencia de sesgo de selección en la investigación. Por otro lado, el diseño de estudio (transversal) no permite establecer relaciones causales, solamente factores asociados.

Hubiese sido importante, también, poder comparar la prevalencia encontrada con la prevalencia en la población general de síntomas depresivos medidos con la escala de Zung abreviada; sin embargo, no se han encontrado otros resultados en el país con ese instrumento. Finalmente, se debe recalcar que el presente trabajo no es extrapolable a ninguna otra población puesto que solo se tomaron datos de estudiantes de la Facultad de Ciencias de la Salud de la Universidad Peruana de Ciencias Aplicadas.

Concluimos que existe una alta prevalencia de síntomas depresivos en los estudiantes de la Facultad de Ciencias de la Salud de la UPC, en especial, en la carrera de medicina, lo cual evidencia la necesidad de un programa de apoyo psicológico para mejorar su salud mental, su calidad de vida y, por lo tanto, sus capacidades de aprendizaje y de desarrollo profesional. Los principales factores asociados a la presencia de síntomas depresivos fueron estar conformes con su rendimiento académico y con su situación económica actual como factores de protección y vivir con un familiar externo a la familia nuclear como factor de riesgo.

Se recomienda ampliar el estudio a otras carreras y otras universidades para poder determinar la prevalencia de síntomas depresivos en dichas poblaciones. Además, se puede evaluar otros factores asociados y confirmar si los que hemos hallado se encuentran en otras poblaciones. De esta manera, se podría tener un panorama más claro de esta situación para poder implementar las medidas preventivas necesarias y adecuadas.

\section{AGRADECIMIENTOS}

Al Dr. Percy Mayta-Tristán por su asesoramiento durante el desarrollo de la investigación. A la Dra. 
Claudia Mory Arciniega, por el apoyo durante la recolección de datos; al Dr. Luis Alfonso Díaz, por su disposición y orientación en la elección del instrumento y a María Gracia Pereda por su apoyo en la digitación de la base de datos.

\section{Financiamiento}

Autofinanciado.

\section{Conflictos de Interés}

Los autores declaran no tener conflicto de interés en la publicación del presente articulo.

\section{REFERENCIAS BIBLIOGRÁFICAS}

1. Riveros M. Características sociodemográficas y niveles de depresión en estudiantes ingresantes a la UNMSM a través de la escala CES-D [Tesis de Maestría]. Lima: Facultad de Psicología, Universidad Nacional Mayor de San Marcos; 2004.

2. Hasler G. Pathophysiology of depression: do we have any solid evidence of interest to clinicians? World Psychiatry. 2010;9(3):155-61.

3. Ruíz A, Silva H, Miranda E. Diagnóstico clínico y psicométrico de la depresión en pacientes de medicina general. Rev Med Chile. 2001;129(6):627-33.

4. Agudelo D, Buela G, Donald C. Ansiedad y depresión: el problema de la diferenciación a través de los síntomas. Salud Mental. 2007;30(2):31-41.

5. Perales A, Sogi C, Morales R. Estudio comparativo de salud mental en estudiantes de medicina de dos universidades estatales peruanas. An Fac Med (Lima). 2003;64(4):239-46.

6. Cova F, Alvial W, Aro M, Bonifetti A, Hernández M, Rodríguez C. Problemas de salud mental en estudiantes de la Universidad de Concepción. Ter Psicol. 2007;25(2):105-12.

7. Gaviria S, Rodríguez M, Álvarez T. Calidad de la relación familiar y depresión en estudiantes de medicina de Medellín, Colombia, 2000. Rev Chil Neuro-psiquiatr. 2002;40(1):41-46.
8. Miranda C, Gutierrez J, Bernal F, Escobar C. Prevalencia de depresión en estudiantes de medicina de la U. de Valle. Rev Colomb Psiquiatr. 2000;3(1):251-60.

9. Czernik G, Giménez S, Mora M, Almirón L. Variables sociodemográficas y síntomas de depresión en estudiantes universitarios de Medicina de Corrientes, Argentina. Rev Arg Clin Neuropsiquiatr. 2006;13(2):64-73.

10. Bhugra D. Migration, distress and cultural identity. $\mathrm{Br}$ Med Bull. 2004;69:129-41.

11. Jou H, Fukada H. Stress and social support in mental and physical health of Chinese students in Japan. Psychol Rep. 1997;81(3 Pt2):1303-12.

12. Fuertes C, Martín Laso MA. El inmigrante en la consulta de atención primaria. An Sist Sanit Navar. 2006;29(Supl 1):9-25.

13. Berezon S, Tiburcio M, Medina E. Variables sociodemográficas asociadas a la depresión: Diferencias entre hombres y mujeres que habitan en zonas urbanas de bajos ingresos. Salud Mental. 2005;28(6):33-40.

14. Cova $F$, Melipillán R, Valdivia M, Bravo E, Valenzuela B. Sintomatología depresiva y ansiosa en estudiantes de enseñanza media. Rev Chil Pediatr. 2007;78(2):151-59.

15. Riveros M, Hernández H, Rivera J. Niveles de depresión y ansiedad en estudiantes universitarios de Lima metropolitana. Rev Investig Psicol. 2007;10(1):92-102.

16. Díaz L, Campo A, Rueda GE, Barros J. Propuesta de una versión abreviada de la escala de Zung para depresión. Colombia Med. 2005;36(3):168-72.

17. Castillo-Vilca MJ, Prado-Mendoza CJ, Vega-Dientsmaier JM. Prevalencia de depresión en estudiantes del quinto año de medicina de una universidad privada de Lima. Rev Neuropsiquiatr. 2010;73(1):9-14

18. Osada J, Rojas M, Rosales C, Vega-Dienstmaier JM. Sintomatología ansiosa y depresiva en estudiantes de medicina. Rev Neuropsiquiatr. 2010;73(1):15-19.

19. Matud MP, Guerrero K, Matías RG. Relevancia de las variables sociodemográficas en las diferencias de género en depresión. Int J Clin Health Psychol. 2006;6(1):7-21.

Correspondencia: Reneé Pereyra Elías.

Dirección: Jr. Alonso de Molina \#1387 - Dpto 302. Urbanización Polo Hunt, Monterrico, Lima, Perú.

Teléfono: (511) 345-2220 / (51) 981-213-381.

Correo electrónico: refranpe7@hotmail.com

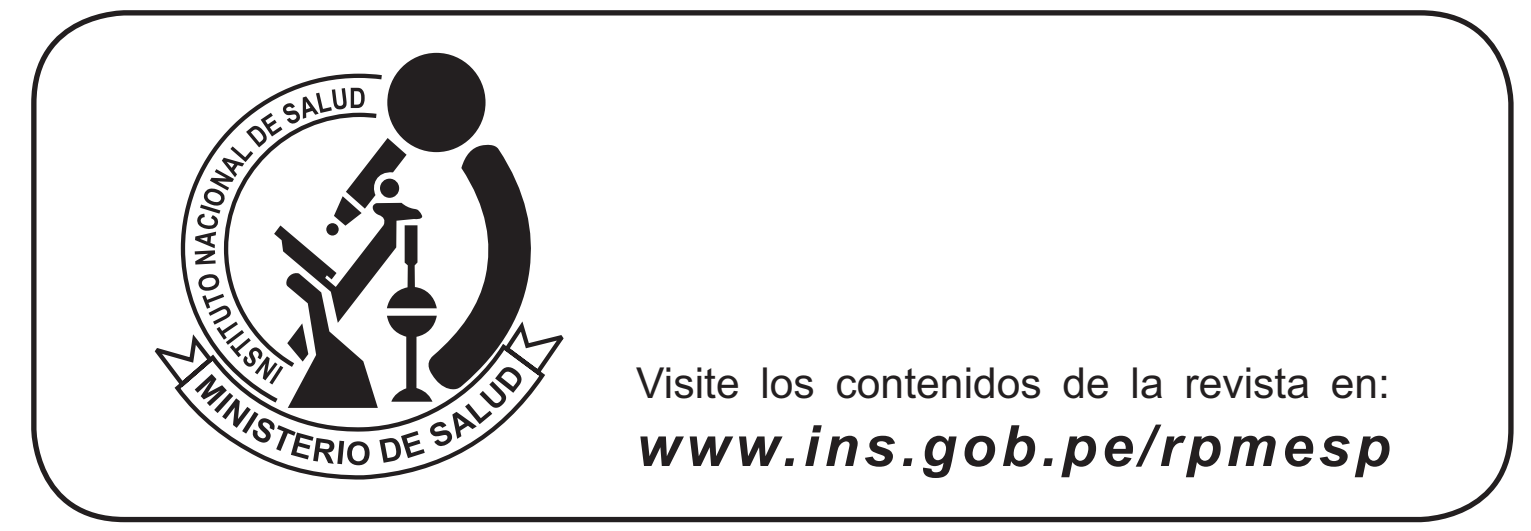

